\title{
COUPLING REQUIREMENTS FOR MULTIPHYSICS PROBLEMS POSED ON TWO DOMAINS*
}

\author{
FATEMEH GHASEMI ${ }^{\dagger}$ AND JAN NORDSTRÖM ${ }^{\dagger}$
}

\begin{abstract}
We consider two hyperbolic systems in first order form of different size posed on two domains. Our ambition is to derive general conditions for when the two systems can and cannot be coupled. The adjoint equations are derived and well-posedness of the primal and dual problems is discussed. By applying the energy method, interface conditions for the primal and dual problems are derived such that the continuous problems are well posed. The equations are discretized using a high order finite difference method in summation-by-parts form and the interface conditions are imposed weakly in a stable way, using penalty formulations. It is shown that one specific choice of penalty matrices leads to a dual consistent scheme. By considering an example, it is shown that the correct physical coupling conditions are contained in the set of well posed coupling conditions. It is also shown that dual consistency leads to superconverging functionals and reduced stiffness.
\end{abstract}

Key words. well posed problems, high order finite differences, stability, summation-by-parts, weak interface conditions, dual consistency, stiffness, superconvergence

AMS subject classifications. 35L50, 65N06, 65N12, 74F99

DOI. $10.1137 / 16 \mathrm{M} 1087710$

1. Introduction. Roughly speaking, a well posed initial boundary value problem requires that a unique solution estimated in terms of data exists. The most common procedure for showing well-posedness is the so-called energy method. In this method, one multiplies the governing partial differential equations (PDEs) with the solution, integrates by parts, and imposes a minimal number of boundary conditions, such that an energy estimate is obtained $[9,15,25]$. This procedure leads to a well posed problem. However, for the coupling of multiphysics problems [8, 17, 22, 23, 33, 27] at an interface, this procedure is less well known. The reason for that is the somewhat more unclear nature of coupling conditions compared to boundary conditions $[12,17,22,23,26]$, even though there are similarities. Well-posedness, especially related to the coupling of multiphysics problems, is discussed in $[19,20,23,9,15]$.

First, accuracy relations must exist such that a combination of variables for one set of PDEs at the interface is equal to another combination of variables for the other set. These sets of variables must also have the same physical properties. For an illustrating example, see [23]. Second, the number of accuracy relations must fit both problems. Too many conditions ruin existence and too few ruin uniqueness. If the number of accuracy relations is too low, additional conditions requiring external data must be added. If the number of accuracy relations is too high, only a subset can be used. Third, the accuracy relations must be such that no artificial growth or decay is generated.

Sensitivity analysis for systems of PDEs is often used in optimization, parameter estimation, model simplification, data assimilation, optimal control, uncertainty analysis, and experimental design [13,14]. For problems involving a large number of sensitivity parameters, the adjoint method is the most efficient [16]. In this paper,

${ }^{*}$ Received by the editors August 3, 2016; accepted for publication (in revised form) June 27, 2017; published electronically November 21, 2017.

http://www.siam.org/journals/sinum/55-6/M108771.html

${ }^{\dagger}$ Computational Mathematics, Department of Mathematics, Linköping University, SE-581 83 Linköping, Sweden (fatemeh.ghasemi@liu.se, jan.nordstrom@liu.se).

2885 
we derive the adjoint equations and interface conditions for these types of problems, such that the adjoint (or dual) problem is also well posed and stable.

It has been shown that dual consistent summation-by-parts (SBP) discretizations lead to superconvergent functionals [10]. Additionally, in [2, 3, 4], it was shown that the dual boundary conditions depend on the primal boundary conditions and vice versa. The fact that the dual and primal boundary conditions are mutually dependent leads to new types of dual consistent boundary conditions. We will use the same technique and derive dual consistent interface conditions. The main goal of this paper is to derive well posed dual interface conditions and a stable dual consistent scheme.

The rest of the paper proceeds as follows. In section 2, the interface conditions, well-posedness, and stability of the primal problem are derived. The dual problem, its interface conditions, well-posedness, and stability are presented in section 3 . We analyze dual consistency for the discrete problems in section 4 . Section 5 contains an example of the theory developed here and the numerical results for both the primal and dual problems. Finally, in section 6 we summarize and draw conclusions.

2. The primal problem. A typical example of problems we consider in this paper is the coupling of the linearized symmetrized Euler equations for fluid flow

$$
u_{t}+A u_{x}=0, \quad-1<x<0,
$$

to the wave equation for the deformation of a solid,

$$
v_{t t}-c_{s}^{2} v_{x x}=0, \quad 0<x<1 .
$$

The matrix and vector in (1) are

$$
A=\left[\begin{array}{ccc}
\bar{w} & c_{f} / \sqrt{\gamma} & 0 \\
c_{f} / \sqrt{\gamma} & \bar{w} & c_{f} \sqrt{\frac{\gamma-1}{\gamma}} \\
0 & c_{f} \sqrt{\frac{\gamma-1}{\gamma}} & \bar{w}
\end{array}\right] \text { and } u=\left[\frac{c_{f} \rho}{\sqrt{\gamma} \bar{\rho}}, w, \frac{-c_{f} \rho}{\bar{\rho} \sqrt{\gamma(\gamma-1)}}+\frac{p \sqrt{\gamma /(\gamma-1)}}{\bar{\rho} c_{f}}\right]^{T} \text {, }
$$

respectively. In (3), $\rho, w, p$, and $c_{f}$ are respectively the density, the velocity, the pressure perturbation, and the speed of sound. The solution we have linearized around is denoted by overbars and $\gamma$ is the ratio of specific heats [1]. In (2), $v$ is the displacement and $c_{s}=\sqrt{E / \rho_{s}}$ is wave speed, where $E$ is the elastic modulus and $\rho_{s}$ is the density of solid. The subscripts $f$ and $s$ refer to the values from the fluid and the solid, respectively.

The second order equation (2) can be rewritten as a first order system given by

$$
U_{t}+B U_{x}=0, \quad B=\left[\begin{array}{cc}
0 & c_{s} \\
c_{s} & 0
\end{array}\right]
$$

where $U=\left[v_{t},-c_{s} v_{x}\right]^{T}$ and $v_{t}$ and $v_{x}$ are the velocity and the stress, respectively.

The possibility to couple problems like (1) and (2) (or (4)) is the main topic in this paper.

2.1. The general formulation. We will consider interface conditions for the hyperbolic systems

$$
\begin{aligned}
u_{t}+A u_{x} & =0, & -1<x<0, & t>0, \\
u(x, 0) & =f(x), & -1<x<0, & t=0,
\end{aligned}
$$


and

$$
\begin{aligned}
v_{t}+B v_{x} & =0, & 0<x<1, & t>0, \\
v(x, 0) & =g(x), & 0<x<1, & t=0 .
\end{aligned}
$$

In (5)-(6), $A, B$ are $m \times m$ and $n \times n$ symmetric constant matrices, respectively, and $u, v$ are unknown vectors of sizes $m$ and $n$. In general we have $m \neq n$. For clarity we often ignore the boundary conditions at $x= \pm 1$ and focus on the interface conditions at $x=0$.

2.2. The interface conditions and well-posedness. The following definition of well-posedness is used.

DeFINITION 2.1. Consider the coupled problem (5)-(6) with homogeneous boundary conditions and a minimal number of boundary and coupling conditions. The coupled problem is well posed if

$$
\frac{d}{d t}\left(\|u\|_{2}^{2}+\alpha_{c}^{p}\|v\|_{2}^{2}\right) \leq 0,
$$

where $\alpha_{c}^{p}$ is a positive free weight, $\|u\|_{2}^{2}=\int_{-1}^{0} u^{T} u d x$, and $\|v\|_{2}^{2}=\int_{0}^{1} v^{T} v d x$.

We apply the energy method by multiplying (5) and (6) with $u^{T}$ and $v^{T}$, respectively, integrating in space and adding them together. The result is

$$
\frac{d}{d t}\left(\|u\|_{2}^{2}+\alpha_{c}^{p}\|v\|_{2}^{2}\right)=\left.w^{T} E w\right|_{x=0} .
$$

In (7), w $=[u, v]^{T}$ and $E=\operatorname{diag}\left(-A, \alpha_{c}^{p} B\right)$. In the following, all terms are evaluated at $x=0$, unless stated otherwise.

We denote $k=k_{A}^{-}+k_{B}^{+}$as the number of positive eigenvalues of $E$, where $k_{A}^{-}, k_{B}^{+}$ are the number of positive eigenvalues of $-A$ and $B$, respectively. This means that exactly $k$ interface (or accuracy) conditions are required for a well posed problem. Let the interface conditions be described by

$$
C^{p} u=D^{p} v,
$$

where the matrices $C^{p}$ and $D^{p}$ have $k$ linearly independent rows. We will seek matrices $C^{p}$ and $D^{p}$ such that the coupled problem (5)-(6) is well posed according to Definition 2.1. We will use the same technique as in $[9,15]$ to find these matrices.

Since $A$ and $B$ are symmetric, we have

$$
\begin{array}{lll}
A=X \Lambda_{A} X^{T}, & X=\left[X_{+}, X_{-}\right], & \Lambda_{A}=\left[\begin{array}{cc}
\Lambda_{A}^{+} & 0 \\
0 & \Lambda_{A}^{-}
\end{array}\right], \\
B=Y \Lambda_{B} Y^{T}, & Y=\left[Y_{+}, Y_{-}\right], & \Lambda_{B}=\left[\begin{array}{cc}
\Lambda_{B}^{+} & 0 \\
0 & \Lambda_{B}^{-}
\end{array}\right] .
\end{array}
$$

In (9), $X_{+}, X_{-}$and $Y_{+}, Y_{-}$are column matrices containing the eigenvectors related to the positive and negative eigenvalues of $A$ and $B$, respectively. The diagonal block matrices $\Lambda_{A}^{+}, \Lambda_{A}^{-}$and $\Lambda_{B}^{+}, \Lambda_{B}^{-}$contain the positive and negative eigenvalues of $A$ and $B$, respectively. For simplicity (and without restriction) we assume that there are no zero eigenvalues. 
By using (9), the relation (7) can be reformulated as

$$
\begin{aligned}
\frac{d}{d t}\left(\|u\|_{2}^{2}+\alpha_{c}^{p}\|v\|_{2}^{2}\right)= & -\left(X^{T} u\right)^{T} \Lambda_{A}\left(X^{T} u\right)+\alpha_{c}^{p}\left(Y^{T} v\right)^{T} \Lambda_{B}\left(Y^{T} v\right) \\
= & -\left[\left(X_{+}^{T} u\right)^{T} \Lambda_{A}^{+}\left(X_{+}^{T} u\right)+\left(X_{-}^{T} u\right)^{T} \Lambda_{A}^{-}\left(X_{-}^{T} u\right)\right] \\
& +\alpha_{c}^{p}\left[\left(Y_{+}^{T} v\right)^{T} \Lambda_{B}^{+}\left(Y_{+}^{T} v\right)+\left(Y_{-}^{T} v\right)^{T} \Lambda_{B}^{-}\left(Y_{-}^{T} v\right)\right] .
\end{aligned}
$$

We consider interface conditions of the general forms

$$
\begin{aligned}
\left(\Lambda_{A}^{-} X_{-}^{T}-R_{A} X_{+}^{T}\right) u & =\left(T_{A} Y_{-}^{T}+S_{A} Y_{+}^{T}\right) v, \\
\left(\Lambda_{B}^{+} Y_{+}^{T}-R_{B} Y_{-}^{T}\right) v & =\left(T_{B} X_{+}^{T}+S_{B} X_{-}^{T}\right) u .
\end{aligned}
$$

The matrices $R_{A}, T_{A}, S_{A}, R_{B}, T_{B}$, and $S_{B}$ are of sizes $k_{A}^{-} \times k_{A}^{+}, k_{A}^{-} \times k_{B}^{-}, k_{A}^{-} \times k_{B}^{+}, k_{B}^{+} \times$ $k_{B}^{-}, k_{B}^{+} \times k_{A}^{+}$, and $k_{B}^{+} \times k_{A}^{-}$, respectively, where $k_{A}^{+}$and $k_{B}^{-}$are the number of negative eigenvalues of $-A$ and $B$, respectively.

2.2.1. Strongly imposed interface conditions. By inserting (11) into (10) we obtain

$$
\frac{d}{d t}\left(\|u\|_{2}^{2}+\alpha_{c}^{p}\|v\|_{2}^{2}\right)=-\left[\begin{array}{c}
X_{+}^{T} u \\
X_{-}^{T} u \\
Y_{+}^{T} v \\
Y_{-}^{T} v
\end{array}\right]^{T} \underbrace{\left[\begin{array}{cccc}
m_{11} & m_{12} & m_{13} & m_{14} \\
\left(m_{12}\right)^{T} & m_{22} & m_{23} & m_{24} \\
\left(m_{13}\right)^{T} & \left(m_{23}\right)^{T} & m_{33} & m_{34} \\
\left(m_{14}\right)^{T} & \left(m_{24}\right)^{T} & \left(m_{34}\right)^{T} & m_{44}
\end{array}\right]}_{M}\left[\begin{array}{c}
X_{+}^{T} u \\
X_{T}^{T} u \\
Y_{+}^{T} v \\
Y_{-}^{T} v
\end{array}\right],
$$

where

$$
\begin{array}{ll}
m_{11}=\Lambda_{A}^{+}+R_{A}^{T}\left(\Lambda_{A}^{-}\right)^{-1} R_{A}-\alpha_{c}^{p} T_{B}^{T}\left(\Lambda_{B}^{+}\right)^{-1} T_{B}, & m_{23}=0, \\
m_{12}=\alpha_{c}^{p} T_{B}^{T}\left(\Lambda_{B}\right)^{-1} S_{B}, & m_{24}=\alpha_{c}^{p} S_{B}^{T}\left(\Lambda_{B}^{+}\right) R_{B}, \\
m_{13}=R_{A}^{T}\left(\Lambda_{A}^{-}\right)^{-1} S_{A}, & m_{33}=S_{A}^{T}\left(\Lambda_{A}^{-}\right)^{-1} S_{A}, \\
m_{14}=R_{A}^{T}\left(\Lambda_{A}^{-}\right)^{-1} T_{A}-\alpha_{c}^{p} T_{B}^{T}\left(\Lambda_{B}^{+}\right)^{-1} R_{B}, & m_{34}=S_{A}^{T}\left(\Lambda_{A}^{-}\right)^{-1} T_{A}, \\
m_{22}=-\alpha_{c}^{p} S_{B}^{T}\left(\Lambda_{B}^{+}\right)^{-1} S_{B}, & m_{44}=-\alpha_{c}^{p}\left(\Lambda_{B}^{-}+R_{B}^{T}\left(\Lambda_{B}^{+}\right)^{-1} R_{B}\right) \\
& +T_{A}^{T}\left(\Lambda_{A}^{-}\right)^{-1} T_{A} .
\end{array}
$$

To obtain a well posed problem, the matrices $R_{A}, T_{A}, S_{A}, R_{B}, T_{B}, S_{B}$, and $\alpha_{c}^{p}$ must be chosen such that $M$ in (12) is positive semidefinite.

Proposition 2.2. If $S_{A} \neq 0$ or $S_{B} \neq 0$, then the coupled problem (5)-(6) is not well posed.

Proof. If $S_{A} \neq 0$ or $S_{B} \neq 0$, then the diagonal elements $m_{22}$ or $m_{33}$ are negative and consequently the matrix $M$ in (12) cannot be positive semidefinite.

From now on we consider $S_{A}=S_{B}=0$, which leads to the interface conditions

$$
\left(\Lambda_{A}^{-} X_{-}^{T}-R_{A} X_{+}^{T}\right) u=T_{A} Y_{-}^{T} v, \quad\left(\Lambda_{B}^{+} Y_{+}^{T}-R_{B} Y_{-}^{T}\right) v=T_{B} X_{+}^{T} u
$$

which can be rewritten as

$$
\left(\left[\begin{array}{cc}
-\Lambda_{A}^{-} & 0 \\
0 & \Lambda_{B}^{+}
\end{array}\right]\left[\begin{array}{cc}
-X_{-}^{T} & 0 \\
0 & Y_{+}^{T}
\end{array}\right]-\left[\begin{array}{cc}
-R_{A} & T_{A} \\
-T_{B} & R_{B}
\end{array}\right]\left[\begin{array}{cc}
-X_{+}^{T} & 0 \\
0 & Y_{-}^{T}
\end{array}\right]\right)\left[\begin{array}{l}
u \\
v
\end{array}\right]=0
$$

In (13) (or (14)), we specify the ingoing characteristic variables in terms of the outgoing ones and incoming data.

Copyright (c) by SIAM. Unauthorized reproduction of this article is prohibited. 
Remark 2.3. The form (13) automatically gives the correct number $k=k_{A}^{-}+k_{B}^{+}$ of interface conditions.

For future reference we write the final coupled problem as

$$
\begin{array}{rlrl}
u_{t}+A u_{x} & =0, & -1<x<0, t>0, & \\
u(x, 0) & =f(x), & -1<x<0, t & =0, \\
v_{t}+B v_{x} & =0, & 0<x<1, t>0, \\
v(x, 0) & =g(x), & 0<x<1, t & =0, \\
C^{p} u(0, t) & =D^{p} v(0, t), & x & =0, t>0,
\end{array}
$$

where

$$
C^{p}=\left[\begin{array}{c}
T_{B} X_{+}^{T} \\
\Lambda_{A}^{-} X_{-}^{T}-R_{A} X_{+}^{T}
\end{array}\right], \quad D^{p}=\left[\begin{array}{c}
\Lambda_{B}^{+} Y_{+}^{T}-R_{B} Y_{-}^{T} \\
T_{A} Y_{-}^{T}
\end{array}\right] .
$$

Now, (12) can be rewritten as

$$
\frac{d}{d t}\left(\|u\|_{2}^{2}+\alpha_{c}^{p}\|v\|_{2}^{2}\right)=-\left[\begin{array}{c}
X_{+}^{T} u \\
Y_{-}^{T} v
\end{array}\right]^{T} \underbrace{\left[\begin{array}{cc}
m_{11}^{p} & m_{12}^{p} \\
\left(m_{12}^{p}\right)^{T} & m_{22}^{p}
\end{array}\right]}_{M^{p}}\left[\begin{array}{c}
X_{+}^{T} u \\
Y_{-}^{T} v
\end{array}\right],
$$

where

$$
m_{11}^{p}=m_{11}, \quad m_{12}^{p}=m_{14}, \quad m_{22}^{p}=m_{44} .
$$

In the following proposition, we will find matrices $R_{A}, R_{B}, T_{A}$, and $T_{B}$ such that the coupled problem (15) leads to an energy estimate.

Proposition 2.4. If the matrices $R_{A}, R_{B}, T_{A}, T_{B}$ and the parameter $\alpha_{c}^{p}$ are chosen such that

$$
\begin{aligned}
\Lambda_{A}^{+}+R_{A}^{T}\left(\Lambda_{A}^{-}\right)^{-1} R_{A}>0, & T_{B}^{T}\left(\Lambda_{B}^{+}\right)^{-1} T_{B} \leq\left(\Lambda_{A}^{+}+R_{A}^{T}\left(\Lambda_{A}^{-}\right)^{-1} R_{A}\right) / \alpha_{c}^{p}, \\
\Lambda_{B}^{-}+R_{B}^{T}\left(\Lambda_{B}^{+}\right)^{-1} R_{B}<0, & T_{A}^{T}\left(\Lambda_{A}^{-}\right)^{-1} T_{A} \geq \alpha_{c}^{p}\left(\Lambda_{B}^{-}+R_{B}^{T}\left(\Lambda_{B}^{+}\right)^{-1} R_{B}\right),
\end{aligned}
$$

and

$$
R_{A}^{T}\left(\Lambda_{A}^{-}\right)^{-1} T_{A}=\alpha_{c}^{p} T_{B}^{T}\left(\Lambda_{B}^{+}\right)^{-1} R_{B},
$$

then, the matrix $M^{p}$ in (16) is positive semidefinite and an energy estimate is obtained for (15).

Proof. Since (19) is satisfied, $m_{12}^{p}=0$ and the matrix $M^{p}$ has the form

$$
\left[\begin{array}{cc}
m_{11}^{p} & 0 \\
0 & m_{22}^{p}
\end{array}\right]
$$

which is positive semidefinite due to the conditions in (18).

Remark 2.5. Note that if $m_{11}^{p}=0$ or $m_{22}^{p}=0$ and $m_{12}^{p} \neq 0$, the coupling cannot be done.

Copyright $@$ by SIAM. Unauthorized reproduction of this article is prohibited. 
Remark 2.6. By replacing $x$ with $-x$ for $-1 \leq x \leq 0$, the coupled problem (5)-(6) can be considered as the initial boundary value problem

$$
\begin{aligned}
w_{t}+G w_{x} & =0, \quad 0 \leq x \leq 1, \\
w(x, 0) & =[f(x), g(x)]^{T},
\end{aligned}
$$

where $w=[u, v]^{T}$ and $G=\operatorname{diag}(-A, B)$. Boundary conditions for the system (20) at $x=0$ will be the interface conditions for the coupled problem (5)-(6). By applying the same technique as in $[9,15]$, we can derive the boundary conditions

$$
\left(\Lambda_{G}^{+} Z_{+}^{T}-R Z_{-}^{T}\right) w=0, \quad Z_{+}=\left[\begin{array}{cc}
-X_{-} & 0 \\
0 & Y_{+}
\end{array}\right], \quad Z_{-}=\left[\begin{array}{cc}
-X_{+} & 0 \\
0 & Y_{-}
\end{array}\right],
$$

at $x=0$. In $(21), \Lambda_{G}^{+}=\operatorname{diag}\left(-\Lambda_{A}^{-}, \Lambda_{B}^{+}\right)$and $R$ is a $2 \times 2$ block matrix. If we choose the matrix $R$ as

$$
R=\left[\begin{array}{ll}
-R_{A} & T_{A} \\
-T_{B} & R_{B}
\end{array}\right]
$$

then the conditions (14) and (21) are equivalent.

2.2.2. Weakly imposed interface conditions. In order to prepare for the numerical approximation, we impose the interface condition (8) weakly. The result is

$$
\begin{aligned}
\frac{d}{d t}\left(\|u\|_{2}^{2}+\alpha_{c}^{p}\|v\|_{2}^{2}\right)= & -u^{T} A u+\alpha_{c}^{p} v^{T} B v \\
& +u^{T} \Sigma_{L}\left(C^{p} u-D^{p} v\right)+\left(u^{T} \Sigma_{L}^{p}\left(C^{p} u-D^{p} v\right)\right)^{T} \\
& +\alpha_{c}^{p}\left[v^{T} \Sigma_{R}\left(D^{p} v-C^{p} u\right)+\left(v^{T} \Sigma_{R}\left(D^{p} v-C^{p} u\right)\right)^{T}\right],
\end{aligned}
$$

where $\Sigma_{L}$ and $\Sigma_{R}$ are penalty matrices of sizes $m \times k$ and $n \times k$, respectively. The relation (23) can be rewritten in matrix form as

$$
\frac{d}{d t}\left(\|u\|_{2}^{2}+\alpha_{c}^{p}\|v\|_{2}^{2}\right)=-\left[\begin{array}{c}
u \\
v
\end{array}\right]^{T} \underbrace{\left[\begin{array}{cc}
n_{11}^{p} & n_{12}^{p} \\
\left(n_{12}^{p}\right)^{T} & n_{22}^{p}
\end{array}\right]}_{N^{p}}\left[\begin{array}{l}
u \\
v
\end{array}\right],
$$

where

$$
\begin{aligned}
& n_{11}^{p}=A-\Sigma_{L} C^{p}-\left(\Sigma_{L} C^{p}\right)^{T}, \quad n_{12}^{p}=\Sigma_{L} D^{p}+\alpha_{c}^{p}\left(\Sigma_{R} C^{p}\right)^{T}, \\
& n_{22}^{p}=-\alpha_{c}^{p}\left(B+\Sigma_{R} D^{p}+\left(\Sigma_{R} D^{p}\right)^{T}\right) .
\end{aligned}
$$

Next, we must find the matrices $\Sigma_{L}$ and $\Sigma_{R}$ such that the matrix $N^{p}$ in (24) is positive semidefinite. Let

$$
\tilde{N}^{p}=\left[\begin{array}{cc}
I & E^{p} \\
0 & I
\end{array}\right]^{T} N^{p}\left[\begin{array}{cc}
I & E^{p} \\
0 & I
\end{array}\right]
$$

and note that if $\tilde{N}^{p}$ is positive semidefinite, so is $N^{p}$. The choice $E^{p}=-\left(n_{11}^{p}\right)^{-1} n_{12}^{p}$ gives

$$
\tilde{N}^{p}=\left[\begin{array}{cc}
n_{11}^{p} & 0 \\
0 & -\left(n_{12}^{p}\right)^{T}\left(n_{11}^{p}\right)^{-1} n_{12}^{p}+n_{22}^{p}
\end{array}\right]
$$

Copyright (c) by SIAM. Unauthorized reproduction of this article is prohibited. 
By choosing $\Sigma_{L}$ and $\Sigma_{R}$ such that

$$
n_{11}^{p}>0, \quad-\left(n_{12}^{p}\right)^{T}\left(n_{11}^{p}\right)^{-1} n_{12}^{p}+n_{22}^{p} \geq 0
$$

then the right-hand side of (24) is bounded.

The above procedure is formalized as follows.

Proposition 2.7. By choosing the penalty matrices $\Sigma_{L}$ and $\Sigma_{R}$ such that (26) is satisfied, the coupled problem (15) is well posed.

In the following proposition, we specify special choices of matrices $\Sigma_{L}$ and $\Sigma_{R}$, which lead to well-posedness. In section 4 , it will be shown that the discrete approximation of (15) is dual consistent for these specific choices.

Proposition 2.8. If the matrices $R_{A}, T_{A}, R_{B}, T_{B}$, and $\alpha_{c}^{p}$ satisfy (18) and (19), then the matrices

$$
\Sigma_{L}=X\left[\begin{array}{cc}
0 & 0 \\
0 & I_{A}^{-}
\end{array}\right], \quad \Sigma_{R}=-Y\left[\begin{array}{cc}
I_{B}^{+} & 0 \\
0 & 0
\end{array}\right]
$$

guarantee that (26) is satisfied. In (27), $I_{A}^{-}$and $I_{B}^{+}$are identity matrices of size $k_{A}^{-}$ and $k_{B}^{+}$, respectively.

Proof. By inserting the matrices $\Sigma_{L}$ and $\Sigma_{R}$ into (25) we obtain

$$
N^{p}=\left[\begin{array}{c}
X_{+}^{T} \\
X_{-}^{T} \\
Y_{+}^{T} \\
Y_{-}^{T}
\end{array}\right]^{T} \bar{N}^{p}\left[\begin{array}{c}
X_{+}^{T} \\
X_{-}^{T} \\
Y_{+}^{T} \\
Y_{-}^{T}
\end{array}\right] \text {, where } \bar{N}^{p}=\left[\begin{array}{cccc}
\Lambda_{A}^{+} & R_{A}^{T} & -\alpha_{c}^{p} T_{B}^{T} & 0 \\
R_{A} & -\Lambda_{A}^{-} & 0 & T_{A} \\
-\alpha_{c}^{p} T_{B} & 0 & \alpha_{c}^{p} \Lambda_{B}^{+} & -\alpha_{c}^{p} R_{B} \\
0 & T_{A}^{T} & -\alpha_{c}^{p} R_{B}^{T} & -\alpha_{c}^{p} \Lambda_{B}^{-}
\end{array}\right] .
$$

Note that the interface terms in (16) can be rewritten as

$$
-\left[\begin{array}{c}
X_{+}^{T} u \\
X_{-}^{T} u \\
Y_{+}^{T} v \\
Y_{-}^{T} v
\end{array}\right]^{T} \tilde{M}^{p}\left[\begin{array}{c}
X_{+}^{T} u \\
X_{-}^{T} u \\
Y_{+}^{T} v \\
Y_{-}^{T} v
\end{array}\right] \text {, where } \tilde{M}^{p}=\left[\begin{array}{cccc}
m_{11}^{p} & 0 & 0 & m_{12}^{p} \\
0 & 0 & 0 & 0 \\
0 & 0 & 0 & 0 \\
\left(m_{12}^{p}\right)^{T} & 0 & 0 & m_{22}^{p}
\end{array}\right]
$$

The matrix $\tilde{M}^{p}$ is positive semidefinite due to the result obtained for the strong interface conditions in Proposition 2.4. To take advantage of this, we rewrite the matrix $\bar{N}^{p}$ as $\bar{N}^{p}=\tilde{M}^{p}+\hat{N}^{p}$, where

$$
\begin{gathered}
\hat{N}^{p}=\left[\begin{array}{cccc}
-R_{A}^{T}\left(\Lambda_{A}^{-}\right)^{-1} R_{A} & R_{A}^{T} & 0 & -R_{A}^{T}\left(\Lambda_{A}^{-}\right)^{-1} T_{A} \\
R_{A} & -\Lambda_{A}^{-} & 0 & T_{A} \\
0 & 0 & 0 & 0 \\
-T_{A}^{T}\left(\Lambda_{A}^{-}\right)^{-1} R_{A} & T_{A}^{T} & 0 & -T_{A}^{T}\left(\Lambda_{A}^{-}\right)^{-1} T_{A}
\end{array}\right] \\
+\alpha_{c}^{p}\left[\begin{array}{cccc}
+T_{B}^{T}\left(\Lambda_{B}^{+}\right)^{-1} T_{B} & 0 & -T_{B}^{T} & +T_{B}^{T}\left(\Lambda_{B}^{+}\right)^{-1} R_{B} \\
0 & 0 & 0 & 0 \\
-T_{B} & 0 & \Lambda_{B}^{+} & -R_{B} \\
+R_{B}^{T}\left(\Lambda_{B}^{+}\right)^{-1} T_{B} & 0 & -R_{B}^{T} & +R_{B}^{T}\left(\Lambda_{B}^{+}\right)^{-1} R_{B}
\end{array}\right] .
\end{gathered}
$$

The matrix $\hat{N}^{p}$ which is due to the use of weak interface conditions can be rewritten as

$$
\hat{N}^{p}=L_{A}\left(C_{A} \otimes\left(\Lambda_{A}^{-}\right)^{-1}\right) L_{A}^{T}+\alpha_{c}^{p} L_{B}\left(C_{B} \otimes\left(\Lambda_{B}^{+}\right)^{-1}\right) L_{B}^{T},
$$


where

$$
\begin{array}{rlr}
L_{A} & =\left[\begin{array}{cccc}
R_{A} & 0 & 0 & 0 \\
0 & \Lambda_{A}^{-} & 0 & 0 \\
0 & 0 & I & 0 \\
0 & 0 & 0 & T_{A}
\end{array}\right], & C_{A}=\left[\begin{array}{rrrr}
-1 & 1 & 0 & -1 \\
1 & -1 & 0 & 1 \\
0 & 0 & 0 & 0 \\
-1 & 1 & 0 & -1
\end{array}\right], \\
L_{B} & =\left[\begin{array}{cccc}
T_{B} & 0 & 0 & 0 \\
0 & I & 0 & 0 \\
0 & 0 & \Lambda_{B}^{+} & 0 \\
0 & 0 & 0 & R_{B}
\end{array}\right], C_{B}=\left[\begin{array}{rrrr}
-1 & 0 & 1 & -1 \\
0 & 0 & 0 & 0 \\
1 & 0 & -1 & 1 \\
-1 & 0 & 1 & -1
\end{array}\right],
\end{array}
$$

and $\otimes$ denotes the Kronecker product [11]. The eigenvalues of the matrices $C_{A}$ and $C_{B}$ are $\{-3,0,0,0\}$, which implies that the matrix $\hat{N}^{p}$ is positive semidefinite.

The difference between the right-hand side in the strong estimate (16) and the right-hand side in the weak estimate $(24)$ is

$$
R=-\left[\begin{array}{c}
X_{+}^{T} u \\
X_{-}^{T} u \\
Y_{+}^{T} v \\
Y_{-}^{T} v
\end{array}\right]^{T} \hat{N}^{p}\left[\begin{array}{c}
X_{+}^{T} u \\
X_{-}^{T} u \\
Y_{+}^{T} v \\
Y_{-}^{T} v
\end{array}\right]
$$

We can expand the term $R$ by using

$$
\begin{aligned}
& C_{A}=X_{A} \Lambda_{C}^{A} X_{A}^{T}, \quad X_{A}=\frac{1}{\sqrt{3}}\left[\begin{array}{rrrr}
1 & -1 & 0 & 1 \\
-1 & 0 & 0 & 1 \\
0 & 0 & 1 & 0 \\
1 & 1 & 0 & 0
\end{array}\right], \Lambda_{C}^{A}=\operatorname{diag}([-3,0,0,0]), \\
& C_{B}=X_{B} \Lambda_{C}^{B} X_{B}^{T}, \quad X_{B}=\frac{1}{\sqrt{3}}\left[\begin{array}{rrrr}
1 & 0 & -1 & 1 \\
0 & 1 & 0 & 0 \\
-1 & 0 & 0 & 1 \\
1 & 0 & 1 & 0
\end{array}\right], \Lambda_{C}^{B}=\Lambda_{C}^{A},
\end{aligned}
$$

and we find that

$$
\begin{aligned}
R & =-\left[\begin{array}{c}
R_{A} X_{+}^{T} u \\
\Lambda_{A}^{-} X_{-}^{T} u \\
Y_{+}^{T} v \\
T_{A} Y_{-}^{T} v
\end{array}\right]^{T}\left(X_{A} \Lambda_{C}^{A} X_{A}^{T} \otimes\left(\Lambda_{A}^{-}\right)^{-1}\right)\left[\begin{array}{c}
R_{A} X_{+}^{T} u \\
\Lambda_{A}^{-} X_{-}^{T} u \\
Y_{+}^{T} v \\
T_{A} Y_{-}^{T} v
\end{array}\right] \\
& -\alpha_{c}^{p}\left[\begin{array}{c}
T_{B} X_{+}^{T} u \\
X_{-}^{T} u \\
\Lambda_{B}^{+} Y_{+}^{T} v \\
R_{B} Y_{-}^{T} v
\end{array}\right]^{T}\left(X_{B} \Lambda_{C}^{B} X_{B}^{T} \otimes\left(\Lambda_{B}^{+}\right)^{-1}\right)\left[\begin{array}{c}
T_{B} X_{+}^{T} u \\
X_{-}^{T} u \\
\Lambda_{B}^{+} Y_{+}^{T} v \\
R_{B} Y_{-}^{T} v
\end{array}\right] \\
& =\left(C^{p} u-D^{p} v\right)^{T} \Lambda^{-1}\left(C^{p} u-D^{p} v\right), \quad \Lambda=\operatorname{diag}\left(\Lambda_{A}^{-},-\alpha_{c}^{p} \Lambda_{B}^{+}\right) .
\end{aligned}
$$

Remark 2.9. The additional seemingly dissipative term $R$ in the weak energy rate is proportional to the interface condition (13) squared and is obviously zero. A nonzero truly dissipative term of the same form will appear in the discrete approximation.

2.3. The semidiscrete primal problem. We now consider finite difference approximations of $(15)$ in SBP form $[6,18,28]$. The interface conditions are implemented using simultaneous approximation terms (SAT) as described in [7, 24, 29, 31].

Copyright $@$ by SIAM. Unauthorized reproduction of this article is prohibited. 
The semidiscrete SBP-SAT formulation of (15) is

$$
\begin{aligned}
& \mathbf{u}_{t}+\left(D_{u} \otimes A\right) \mathbf{u}=P_{u}^{-1} e_{N} \otimes \boldsymbol{\Sigma}_{L}\left(C^{p} u_{N}-D^{p} v_{0}\right), \\
& \mathbf{v}_{t}+\left(D_{v} \otimes B\right) \mathbf{v}=P_{v}^{-1} e_{0} \otimes \boldsymbol{\Sigma}_{R}\left(D^{p} v_{0}-C^{p} u_{N}\right),
\end{aligned}
$$

where the outer boundary conditions are ignored as in the continuous case. The discrete solutions are arranged as

$$
\mathbf{u}=\left(u_{01}, . ., u_{0 m}, . ., u_{N 1}, . ., u_{N m}\right)^{T}, \mathbf{v}=\left(v_{01}, . ., v_{0 n}, . ., v_{M 1}, . ., v_{M n}\right)^{T},
$$

where $u_{N}=\left[u_{N 1}, \ldots, u_{N m}\right]^{T}, v_{0}=\left[v_{01}, \ldots, v_{0 n}\right]^{T}$. In (28), $D_{u, v}=P_{u, v}^{-1} Q_{u, v}$ are difference operators which approximate the first derivative, and the subscripts $u, v$ denote the direction they operate along. The difference operators satisfy $P_{u, v}=$ $P_{u, v}^{T}>0$ and $Q_{u, v}+Q_{u, v}^{T}=\operatorname{diag}([-1,0, \ldots, 0,1]) . \quad e_{N}=(0, \ldots, 1)^{T}$ and $e_{0}=$ $(1, \ldots, 0)^{T}$ are $N+1$ and $M+1$ unit vectors, respectively. The penalty matrices $\boldsymbol{\Sigma}_{L}$ and $\boldsymbol{\Sigma}_{R}$ have the same dimensions as in the continuous case and will be chosen for stability. For a comprehensive review of the SBP-SAT technique, see [32].

2.3.1. Stability conditions at the interface. The discrete energy method is applied to (28) by multiplying the two equations with $\mathbf{u}^{T}\left(P_{u} \otimes I\right), \mathbf{v}^{T}\left(P_{v} \otimes I\right)$, respectively, and adding the result. By defining the discrete norms $\|\mathbf{u}\|_{P_{u} \otimes I}^{2}=$ $\mathbf{u}^{T}\left(P_{u} \otimes I\right) \mathbf{u},\|\mathbf{v}\|_{P_{v} \otimes I}^{2}=\mathbf{v}^{T}\left(P_{v} \otimes I\right) \mathbf{v}$, using the symmetry properties of $A, B$ and the SBP property of $Q_{u, v}$, we obtain

$$
\begin{aligned}
\frac{d}{d t}\left(\|\mathbf{u}\|_{P_{u} \otimes I}^{2}\right. & \left.+\alpha_{d}^{p}\|\mathbf{v}\|_{P_{v} \otimes I}^{2}\right)=-u_{N}^{T} A u_{N}+\alpha_{d}^{p} v_{0}^{T} B v_{0} \\
& +u_{N}^{T} \boldsymbol{\Sigma}_{L}\left(C^{p} u_{N}-D^{p} v_{0}\right)+\left(u_{N}^{T} \boldsymbol{\Sigma}_{L}\left(C^{p} u_{N}-D^{p} v_{0}\right)\right)^{T} \\
& +\alpha_{d}^{p}\left[v_{0}^{T} \boldsymbol{\Sigma}_{R}\left(D^{p} v_{0}-C^{p} u_{N}\right)+\left(v_{0}^{T} \boldsymbol{\Sigma}_{R}\left(D^{p} v_{0}-C^{p} u_{N}\right)\right)^{T}\right],
\end{aligned}
$$

which is similar to the continuous (weak) energy rate in (23).

The relation (29) can be rewritten in the matrix form

$$
\frac{d}{d t}\left(\|\mathbf{u}\|_{P_{u} \otimes I}^{2}+\alpha_{d}^{p}\|\mathbf{v}\|_{P_{v} \otimes I}^{2}\right)=-\left[\begin{array}{c}
u_{N} \\
v_{0}
\end{array}\right]^{T} \mathbf{N}^{p}\left[\begin{array}{c}
u_{N} \\
v_{0}
\end{array}\right]
$$

where we find that $\mathbf{N}^{p}=N^{p}$ in (24) by letting $\alpha_{d}^{p}=\alpha_{c}^{p}$.

We immediately arrive at the following proposition.

Proposition 2.10. By choosing the penalty matrices $\boldsymbol{\Sigma}_{L}$ and $\boldsymbol{\Sigma}_{R}$ such that (26) is satisfied, the semidiscrete approximation (28) of the coupled problem (15) is stable.

Similar to the continuous case, we will specify a set of penalty matrices which leads to stability.

Proposition 2.11. If the matrices $R_{A}, T_{A}, R_{B}, T_{B}$, and $\alpha_{d}^{p}$ satisfy (18), and (19), then the matrices

$$
\boldsymbol{\Sigma}_{L}=X\left[\begin{array}{cc}
0 & 0 \\
0 & I_{A}^{-}
\end{array}\right], \quad \boldsymbol{\Sigma}_{R}=-Y\left[\begin{array}{cc}
I_{B}^{+} & 0 \\
0 & 0
\end{array}\right]
$$

satisfy (26).

Proof. See the proof of Proposition 2.8.

Copyright $@$ by SIAM. Unauthorized reproduction of this article is prohibited. 
Remark 2.12. The derivation in the discrete case is analogous to the continuous one above due to the mimicking properties of the SBP-SAT technique. In fact, the interface conditions and penalty matrices are already derived in the continuous setting; see [21] for more details on this technique.

The discrete energy rate (30) is similar to the continuous one with the additional term

$$
\mathbf{R}=\left(C^{p} u_{N}-D^{p} v_{0}\right)^{T}(\Lambda)^{-1}\left(C^{p} u_{N}-D^{p} v_{0}\right), \quad \Lambda=\operatorname{diag}\left(\Lambda_{A}^{-},-\alpha_{d}^{p} \Lambda_{B}^{+}\right),
$$

added onto the right-hand side. This term was identically zero in the continuous case but now adds a small amount of dissipation, since $u_{N}$ and $v_{0}$ are approximations of $u(0, t)$ and $v(0, t)$. As we refine the mesh, the term $C^{p} u_{N}-D^{p} v_{0}$ goes to zero and the additional dissipation vanishes.

3. The dual problem. Consider the linear functional

$$
J(w)=(u, h)_{L}+(v, l)_{R},
$$

where $h$ and $l$ are weight functions and $(u, h)_{L}=\int_{-1}^{0} u^{T} h d x,(v, l)_{R}=\int_{0}^{1} v^{T} l d x$. To derive the dual problem, we add forcing functions $f_{L}, f_{R}$ to the right-hand sides of (5)-(6) and seek functions $\phi$ and $\psi$ such that

$$
\int_{0}^{T} J(w) d t=\int_{0}^{T}\left(\phi, f_{L}\right)_{L}+\left(\psi, f_{R}\right)_{R} d t
$$

As an initial step, we observe that

$$
\begin{aligned}
\int_{0}^{T} J(w) d t= & \int_{0}^{T} J(w) d t \\
& -\int_{0}^{T}\left(\phi, u_{t}+A u_{x}-f_{L}\right)_{L} d t-\int_{0}^{T}\left(\psi, v_{t}+B v_{x}-f_{R}\right)_{R} d t .
\end{aligned}
$$

Next, we use integration by parts to get

$$
\begin{aligned}
\int_{0}^{T} J(w) d t= & \int_{0}^{T}\left(\phi, f_{L}\right)_{L}+\left(\psi, f_{R}\right)_{R} d t \\
& +\int_{0}^{T}\left(u, \phi_{t}+A \phi_{x}+h\right)_{L} d t+\int_{0}^{T}\left(v, \psi_{t}+B \psi_{x}+l\right)_{R} d t \\
& -\int_{-1}^{0}\left[\phi^{T} u\right]_{0}^{T} d x-\int_{0}^{1}\left[\psi^{T} v\right]_{0}^{T} d x-\int_{0}^{T}\left(\left[\phi^{T} A u\right]_{-1}^{0}+\left[\psi^{T} B v\right]_{0}^{1}\right) d t .
\end{aligned}
$$

The dual boundary and interface conditions are the minimal number of conditions such that

$$
\int_{0}^{T}\left(\left[\phi^{T} A u\right]_{-1}^{0}+\left[\psi^{T} B v\right]_{0}^{1}\right) d t=0
$$

In the next subsection, explicit interface conditions will be derived. By choosing homogeneous initial and final conditions

$$
u(x, 0)=v(x, 0)=\phi(x, T)=\psi(x, T)=0
$$

for the primal and dual problem, the terms $\int_{-1}^{0}\left[\phi^{T} u\right]_{0}^{T} d x$ and $\int_{0}^{1}\left[\psi^{T} v\right]_{0}^{T} d x$ in (32) vanish, and the following dual equations are obtained:

$$
\begin{aligned}
-\phi_{t}-A \phi_{x}=h, & -1<x<0, \\
-\psi_{t}-B \psi_{x}=l, & 0<x<1 .
\end{aligned}
$$

Copyright $@$ by SIAM. Unauthorized reproduction of this article is prohibited. 
3.1. The dual boundary and interface conditions. The interface conditions (13) grouped together with the interface terms in (33) yield

$$
\begin{aligned}
0=\left(\phi^{T} A u-\psi^{T} B v\right)= & \left(\phi^{T} X_{+} \Lambda_{A}^{+}+\phi^{T} X_{-} R_{A}-\psi^{T} Y_{+} T_{B}\right) X_{+}^{T} u \\
& +\left(\phi^{T} X_{-} T_{A}-\psi^{T} Y_{+} R_{B}-\psi^{T} Y_{-} \Lambda_{B}^{-}\right) Y_{-}^{T} v
\end{aligned}
$$

and arrive at the dual interface conditions

$$
\left(\Lambda_{A}^{+} X_{+}^{T}+R_{A}^{T} X_{-}^{T}\right) \phi=T_{B}^{T} Y_{+}^{T} \psi, \quad\left(\Lambda_{B}^{-} Y_{-}^{T}+R_{B}^{T} Y_{+}^{T}\right) \psi=T_{A}^{T} X_{-}^{T} \phi .
$$

Remark 3.1. Note that the dual interface conditions are given by the primal ones. See $[2,3,4]$ for similar effects regarding boundary conditions.

The dual interface condition (35) can be written more compactly as

$$
C^{a} \phi=D^{a} \psi
$$

where

$$
C^{a}=\left[\begin{array}{c}
\Lambda_{A}^{+} X_{+}^{T}+R_{A}^{T} X_{-}^{T} \\
T_{A}^{T} X_{-}^{T}
\end{array}\right], \quad D^{a}=\left[\begin{array}{c}
T_{B}^{T} Y_{+}^{T} \\
\Lambda_{B}^{-} Y_{-}^{T}+R_{B}^{T} Y_{+}^{T}
\end{array}\right] .
$$

For completeness, the dual boundary conditions must also be determined such that

$$
\left.\phi^{T} A u\right|_{x=-1}=0,\left.\quad \psi^{T} B v\right|_{x=+1}=0 .
$$

To choose dual boundary conditions, the boundary conditions for the primal problem must exist. We consider the following general homogeneous boundary conditions for the primal problem:

$$
\left(\Lambda_{A}^{+} X_{+}^{T}-R_{l} X_{-}^{T}\right) u(-1, t)=0, \quad\left(\Lambda_{B}^{-} Y_{-}^{T}-R_{r} Y_{+}^{T}\right) v(+1, t)=0,
$$

where the matrices $R_{l}$ and $R_{r}$ are such that a well posed primal problem is obtained. This yields

$$
\begin{aligned}
\left.\phi^{T} A u\right|_{x=-1} & =\left.\left(\phi^{T} X_{+} R_{l}+\phi^{T} X_{-} \Lambda_{A}^{-}\right) X_{-}^{T} u\right|_{x=-1}, \\
\left.\psi^{T} B v\right|_{x=+1} & =\left.\left(\psi^{T} Y_{+} \Lambda_{B}^{+}+\psi^{T} Y_{-} R_{r}\right) Y_{+}^{T} v\right|_{x=+1},
\end{aligned}
$$

and we choose the dual boundary conditions

$$
\left(\Lambda_{A}^{-} X_{-}^{T}+R_{l}^{T} X_{+}^{T}\right) \phi(-1, t)=0, \quad\left(\Lambda_{B}^{+} Y_{+}^{T}+R_{r}^{T} Y_{-}^{T}\right) \psi(+1, t)=0,
$$

such that all boundary terms vanish and (37) is satisfied.

The initial conditions for the dual problem are given at time $t=T$. The time transformation $\tau=t-T$ inserted into (34) results in

$$
\begin{array}{rlrl}
\phi_{t}-A \phi_{x} & =h, & -1<x<0, \tau>0, & \\
\phi(x, 0) & =0, & -1<x<0, \tau=0, \\
\psi_{t}-B \psi_{x} & =l, & 0<x<1, \tau>0, & \\
\psi(x, 0) & =0, & 0<x<1, \tau=0, \\
C^{a} \phi(0, \tau) & =D^{a} \psi(0, \tau), & x=0, \tau>0,
\end{array}
$$

which is the final form of the coupled dual problem. Note that the dual interface conditions $C^{a} \phi=D^{a} \psi$, given in (36), specify the ingoing characteristic variables in terms of outgoing ones and incoming data, just as in the primal problem. 
3.1.1. Strongly imposed dual interface conditions. We apply the energy method to (38) and ignore the boundary terms as for the primal problem. By using the dual interface conditions (35), we get

$$
\begin{aligned}
\frac{d}{d \tau}\left(\|\phi\|_{2}^{2}+\alpha_{c}^{a}\|\psi\|_{2}^{2}\right) & =\left.\phi^{T} A \phi\right|_{x=0}-\left.\alpha_{c}^{a} \psi^{T} B \psi\right|_{x=0} \\
& =\left[\begin{array}{c}
X_{-}^{T} \phi \\
Y_{+}^{T} \psi
\end{array}\right]^{T} \underbrace{\left[\begin{array}{cc}
m_{11}^{a} & m_{12}^{a} \\
\left(m_{12}^{a}\right)^{T} & m_{22}^{a}
\end{array}\right]}_{M^{a}}\left[\begin{array}{c}
X_{-}^{T} \phi \\
Y_{+}^{T} \psi
\end{array}\right],
\end{aligned}
$$

where

$$
\begin{aligned}
& m_{11}^{a}=\Lambda_{A}^{-}+R_{A}\left(\Lambda_{A}^{+}\right)^{-1} R_{A}^{T}-\alpha_{c}^{a} T_{A}\left(\Lambda_{B}^{-}\right)^{-1} T_{A}^{T}, \\
& m_{12}^{a}=-R_{A}\left(\Lambda_{A}^{+}\right)^{-1} T_{B}^{T}+\alpha_{c}^{a} T_{A}\left(\Lambda_{B}^{-}\right)^{-1} R_{B}^{T}, \\
& m_{22}^{a}=-\alpha_{c}^{a} \Lambda_{B}^{+}-\alpha_{c}^{a} R_{B}\left(\Lambda_{B}^{-}\right)^{-1} R_{B}^{T}+T_{B}\left(\Lambda_{A}^{+}\right)^{-1} T_{B}^{T},
\end{aligned}
$$

and $\alpha_{c}^{a}$ is a positive weight.

In the following proposition, we will find the matrices $R_{A}, R_{B}, T_{A}$, and $T_{B}$ such that the coupled problem (38) leads to an energy estimate.

Proposition 3.2. If the matrices $R_{A}, R_{B}, T_{A}, T_{B}$ and the parameter $\alpha_{c}^{a}$ are chosen such that

$$
\begin{array}{ll}
\Lambda_{A}^{-}+R_{A}\left(\Lambda_{A}^{+}\right)^{-1} R_{A}^{T}<0, & T_{A}\left(\Lambda_{B}^{-}\right)^{-1} T_{A}^{T} \geq\left(\Lambda_{A}^{-}+R_{A}\left(\Lambda_{A}^{+}\right)^{-1} R_{A}^{T}\right) / \alpha_{c}^{a}, \\
\Lambda_{B}^{+}+R_{B}\left(\Lambda_{B}^{-}\right)^{-1} R_{B}^{T}>0, & T_{B}\left(\Lambda_{A}^{+}\right)^{-1} T_{A}^{T} \leq \alpha_{c}^{a}\left(\Lambda_{B}^{+}+R_{B}\left(\Lambda_{B}^{-}\right)^{-1} R_{B}^{T}\right),
\end{array}
$$

and

$$
R_{A}\left(\Lambda_{A}^{+}\right)^{-1} T_{B}^{T}=\alpha_{c}^{a} T_{A}\left(\Lambda_{B}^{-}\right)^{-1} R_{B}^{T},
$$

then, the matrix $M^{a}$ in (39) is negative semidefinite and an energy estimate is satisfied for (38).

Proof. The proof proceeds as in Proposition 2.4.

3.1.2. Weakly imposed dual interface conditions. As in the primal problem, we impose the interface condition (36) weakly. The result is

$$
\begin{aligned}
\frac{d}{d \tau}\left(\|\phi\|_{2}^{2}+\alpha_{c}^{a}\|\psi\|_{2}^{2}\right)= & \phi^{T} A \phi-\alpha_{c}^{a} \psi^{T} B \psi \\
& +\phi^{T} \Sigma_{L}\left(C^{a} \phi-D^{a} \psi\right)+\left(\phi^{T} \Sigma_{L}\left(C^{a} \phi-D^{a} \psi\right)\right)^{T} \\
& +\alpha_{c}^{a}\left[\psi^{T} \Sigma_{R}\left(D^{a} \psi-C^{a} \phi\right)+\left(\psi^{T} \Sigma_{R}\left(D^{a} \psi-C^{a} \phi\right)\right)^{T}\right],
\end{aligned}
$$

where the penalty matrices $\Sigma_{L}$ and $\Sigma_{R}$ are of size $m \times\left(k_{A}^{+}+k_{B}^{-}\right)$and $n \times\left(k_{A}^{+}+k_{B}^{-}\right)$, respectively.

Remark 3.3. The derivation procedure below for the dual problem is the same as for the primal problem.

The relation (42) can be rewritten in the matrix form

$$
\frac{d}{d \tau}\left(\|\phi\|_{2}^{2}+\alpha_{c}^{a}\|\psi\|_{2}^{2}\right)=\left[\begin{array}{c}
\phi \\
\psi
\end{array}\right]^{T} \underbrace{\left[\begin{array}{cc}
n_{11}^{a} & n_{12}^{a} \\
\left(n_{12}^{a}\right)^{T} & n_{22}^{a}
\end{array}\right]}_{N^{a}}\left[\begin{array}{l}
\phi \\
\psi
\end{array}\right],
$$

Copyright (c) by SIAM. Unauthorized reproduction of this article is prohibited. 
where

$$
\begin{aligned}
& n_{11}^{a}=A+\Sigma_{L} C^{a}+\left(\Sigma_{L} C^{a}\right)^{T}, \quad n_{12}^{a}=-\Sigma_{L} D^{a}-\alpha_{c}^{a}\left(\Sigma_{R} C^{a}\right)^{T}, \\
& n_{22}^{a}=-\alpha_{c}^{a}\left(B-\Sigma_{R} D^{a}-\left(\Sigma_{R} D^{a}\right)^{T}\right) .
\end{aligned}
$$

By using the rotation technique, as in the primal problem, we arrive at the following.

Proposition 3.4. The choice of penalty matrices $\Sigma_{L}$ and $\Sigma_{R}$ such that

$$
n_{11}^{a}<0, \quad-\left(n_{12}^{a}\right)^{T}\left(n_{11}^{a}\right)^{-1} n_{12}^{a}+n_{22}^{a} \leq 0
$$

holds leads to well-posedness of the coupled problem (38).

As in the primal problem, we specify special choices of matrices $\Sigma_{L}$ and $\Sigma_{R}$, which lead to well-posedness.

Proposition 3.5. If the matrices $R_{A}, T_{A}, R_{B}, T_{B}$, and $\alpha_{c}^{a}$ satisfy (40) and (41), then the matrices

$$
\Sigma_{L}=-X\left[\begin{array}{cc}
I_{A}^{+} & 0 \\
0 & 0
\end{array}\right], \quad \Sigma_{R}=Y\left[\begin{array}{cc}
0 & 0 \\
0 & I_{B}^{-}
\end{array}\right]
$$

guarantee that (44) holds. In (45), $I_{A}^{+}$and $I_{B}^{-}$are identity matrices of size $k_{A}^{+}$and $k_{B}^{-}$, respectively.

Proof. See the proof of Proposition 2.7.

3.2. The semidiscrete approximation of the dual problem. The corresponding semidiscrete SBP-SAT formulation of (38) is

$$
\begin{aligned}
\boldsymbol{\phi}_{\tau}-\left(D_{u} \otimes A\right) \boldsymbol{\phi} & =P_{u}^{-1} e_{N} \otimes \boldsymbol{\Sigma}_{L}\left(C^{a} \phi_{N}-D^{a} \psi_{0}\right), \\
\boldsymbol{\psi}_{\tau}-\left(D_{v} \otimes B\right) \boldsymbol{\psi} & =P_{v}^{-1} e_{0} \otimes \boldsymbol{\Sigma}_{R}\left(D^{a} \psi_{0}-C^{a} \phi_{N}\right),
\end{aligned}
$$

where the outer boundary conditions are ignored as in the primal case. The vectors $\phi_{N}$ and $\psi_{0}$ are arranged as $\phi_{N}=\left[\phi_{N 1}, \ldots, \phi_{N m}\right]^{T}, \psi_{0}=\left[\psi_{01}, \ldots, \psi_{0 n}\right]^{T}$. The penalty matrices $\boldsymbol{\Sigma}_{L}$ and $\boldsymbol{\Sigma}_{R}$ have the same dimensions as in the continuous case.

3.2.1. Stability conditions at the interface. Applying the discrete energy method to (46) leads to

$$
\begin{aligned}
& \frac{d}{d \tau}\left(\|\boldsymbol{\phi}\|_{P_{u} \otimes I}^{2}+\alpha_{d}^{a}\|\boldsymbol{\psi}\|_{P_{v} \otimes I}^{2}\right)=\phi_{N}^{T} A \phi_{N}-\alpha_{d}^{a} \psi_{0}^{T} B \psi_{0} \\
& \quad+\phi_{N}^{T} \boldsymbol{\Sigma}_{L}\left(C^{a} \phi_{N}-D^{a} \psi_{0}\right)+\left(\phi_{N}^{T} \boldsymbol{\Sigma}_{L}\left(C^{a} \phi_{N}-D^{a} \psi_{0}\right)\right)^{T} \\
& \quad+\alpha_{d}^{a}\left[\psi_{0}^{T} \boldsymbol{\Sigma}_{R}\left(D^{a} \psi_{0}-C^{a} \phi_{N}\right)+\left(\psi_{0}^{T} \boldsymbol{\Sigma}_{R}\left(D^{a} \psi_{0}-C^{a} \phi_{N}\right)\right)^{T}\right],
\end{aligned}
$$

which is similar to the continuous case (42). The relation (47) can be rewritten in the matrix form

$$
\frac{d}{d t}\left(\|\mathbf{u}\|_{P_{u} \otimes I}^{2}+\alpha_{d}^{p}\|\mathbf{v}\|_{P_{v} \otimes I}^{2}\right)=\left[\begin{array}{c}
\phi_{N} \\
\psi_{0}
\end{array}\right]^{T} \mathbf{N}^{a}\left[\begin{array}{c}
\phi_{N} \\
\psi_{0}
\end{array}\right]
$$

where we find that $\mathbf{N}^{a}=N^{a}$ in (43) by letting $\alpha_{a}^{p}=\alpha_{c}^{a}$.

We immediately arrive at the following proposition.

Copyright $@$ by SIAM. Unauthorized reproduction of this article is prohibited. 
Proposition 3.6. By choosing the penalty matrices $\boldsymbol{\Sigma}_{L}$ and $\boldsymbol{\Sigma}_{R}$ such that (44) is satisfied, the semidiscrete approximation (46) of the coupled problem (38) is stable.

Similar to the continuous case, we will specify a set of penalty matrices which leads to stability.

Proposition 3.7. If the matrices $R_{A}, T_{A}, R_{B}, T_{B}$, and $\alpha_{d}^{a}$ satisfy (40) and (41), then the matrices

$$
\boldsymbol{\Sigma}_{L}=-X\left[\begin{array}{cc}
I_{A}^{+} & 0 \\
0 & 0
\end{array}\right], \quad \boldsymbol{\Sigma}_{R}=Y\left[\begin{array}{cc}
0 & 0 \\
0 & I_{B}^{-}
\end{array}\right]
$$

guarantee that (44) holds and that (46) is stable.

Proof. See the proof of Proposition 2.8.

Remark 3.8. Just as in the primal problem, the penalty matrices in the discrete case are the same as in the continuous one, due to the similarity in analysis of the continuous and discrete problem.

4. Dual consistency. To investigate dual consistency, we rewrite (28) and (46) into the following form:

$$
P \mathbf{w}_{t}+L^{p} \mathbf{w}=P F, \quad P \boldsymbol{\theta}_{\tau}+L^{a} \boldsymbol{\theta}=P H,
$$

where

$$
\begin{aligned}
& L^{p}=+\left[\begin{array}{cc}
Q_{u} \otimes A & 0 \\
0 & Q_{v} \otimes B
\end{array}\right]+\left[\begin{array}{ccccc}
0 & & & & \\
& \ddots & & & \\
& -\boldsymbol{\Sigma}_{L} C^{p} & \boldsymbol{\Sigma}_{L} D^{p} & & \\
& \boldsymbol{\Sigma}_{R} C^{p} & -\boldsymbol{\Sigma}_{R} D^{p} & & \\
& & & \ddots & \\
& & & & 0
\end{array}\right], \\
& L^{a}=-\left[\begin{array}{cc}
Q_{u} \otimes A & 0 \\
0 & Q_{v} \otimes B
\end{array}\right]+\left[\begin{array}{ccccc}
0 & & & & \\
& \ddots & & & \\
& -\Sigma_{L} C^{a} & \Sigma_{L} D^{a} & & \\
& \Sigma_{R} C^{a} & -\Sigma_{R} D^{a} & & \\
& & & \ddots & \\
& & & & 0
\end{array}\right] \text {, }
\end{aligned}
$$

and

$$
P^{-1}=\left[\begin{array}{cc}
P_{u}^{-1} \otimes I_{m} & 0 \\
0 & P_{v}^{-1} \otimes I_{n}
\end{array}\right] .
$$

In (49), w $=[\mathbf{u}, \mathbf{v}]^{T}, \boldsymbol{\theta}=[\boldsymbol{\phi}, \boldsymbol{\psi}]^{T}$, and $F=\left[F_{L}, F_{R}\right], H=\left[H_{L}, H_{R}\right]$, where $F_{L}, F_{R}, H_{L}$ and $H_{R}$ are the discrete forms of $f_{L}, f_{R}, h_{L}$, and $h_{R}$, respectively.

Remark 4.1. The similarity between the second matrix in $L^{p}$ and $L^{a}$ is due to the similarity of the right-hand sides in (28) and (46).

The schemes in (49) are dual consistent $[2,10]$ if

$$
\left(L^{p}\right)^{T}=L^{a} .
$$

Copyright (c) by SIAM. Unauthorized reproduction of this article is prohibited. 
Since $Q_{u, v}+Q_{u, v}^{T}=E_{N}-E_{0}$, the requirement (50) leads to the condition

$$
\left[\begin{array}{cc}
A-\left(C^{p}\right)^{T} \boldsymbol{\Sigma}_{L}^{T} & \left(C^{p}\right)^{T} \boldsymbol{\Sigma}_{R}^{T} \\
\left(D^{p}\right)^{T} \boldsymbol{\Sigma}_{L}^{T} & -\left(D^{p}\right)^{T} \boldsymbol{\Sigma}_{R}^{T}-B
\end{array}\right]=\left[\begin{array}{cc}
-\boldsymbol{\Sigma}_{L} C^{a} & \boldsymbol{\Sigma}_{L} D^{a} \\
\boldsymbol{\Sigma}_{R} C^{a} & -\boldsymbol{\Sigma}_{R} D^{a}
\end{array}\right]
$$

We find the following.

Proposition 4.2. If the penalty matrices $\boldsymbol{\Sigma}_{L, R}$ and $\boldsymbol{\Sigma}_{L, R}$ are chosen as in (31) and (48), respectively, then the SBP-SAT discretization of the coupled problem (15) is dual consistent

Proof. A direct insertion of (31) and (48) into (51) yields the result.

Remark 4.3. By choosing the penalty matrices such that (26) is satisfied but (51) is not, the semidiscrete approximation (28) is stable but dual inconsistent.

Remark 4.4. We summarize what has been done so far below:

- Well posed interface conditions for the primal problem have been derived. The interface conditions are imposed and penalty matrices are obtained such that the continuous problem is well posed. The penalty matrices for the continuous problem lead directly to a stable discrete primal problem.

- By using the same strategy as in the primal problem, the interface conditions and penalty matrices for the continuous coupled dual problem are derived. These matrices lead to a stable discrete dual problem. The dual interface condition is determined by the primal interface condition.

- A specific set of penalty matrices for the primal and dual problem can be chosen such that the discrete problems are stable and the primal discrete problem is dual consistent.

5. The physical example. In this section, we consider the physical example in section 2. We will investigate if the mathematical theory derived earlier will provide us with the physical coupling conditions. The physical interface conditions come from mechanical principles: (1) continuity and (2) force balance (Newton's second law). According to the first principle the fluid and solid velocities must match at the interface; otherwise the fluid will be detached from the solid. This means that one of the interface conditions is

$$
w=v_{t} .
$$

The fluid pushes on the solid with a traction $p$ and the solid pushes back with an equal and opposite traction, which is called $\sigma$, and we get $\sigma=-p$. For a linear elastic material, according to Hooke's law, we have $\sigma=E v_{x}$, which implies that the second interface condition is

$$
p=-E v_{x} .
$$

Now, we apply the mathematical approach. The matrices $A$ and $B$ in (3) and (4) can be written as

$$
A=X_{+} \Lambda_{A}^{+} X_{+}^{T}+X_{-} \Lambda_{A} X_{-}^{T}, \quad B=Y_{+} \Lambda_{B}^{+} Y_{+}^{T}+Y_{-} \Lambda_{B} Y_{-}^{T},
$$

where

$$
\begin{array}{rlrl}
X_{+} & =\frac{1}{\sqrt{2}}\left[\begin{array}{ccc}
\sqrt{\frac{2(\gamma-1)}{\gamma}} & 0 & -\sqrt{2 / \gamma} \\
\sqrt{1 / \gamma} & 1 & \sqrt{\frac{(\gamma-1)}{\gamma}}
\end{array}\right]^{T}, & X_{-}=\frac{1}{\sqrt{2}}[\sqrt{1 / \gamma},-1, \sqrt{(\gamma-1) / \gamma}]^{T} \\
Y_{+}=\frac{1}{\sqrt{2}}[1,1]^{T}, & Y_{-}=\frac{1}{\sqrt{2}}[1,-1]^{T},
\end{array}
$$


and

$$
\Lambda_{A}^{+}=\left[\begin{array}{cc}
\bar{w} & 0 \\
0 & \bar{w}+c_{f}
\end{array}\right], \quad \Lambda_{A}^{-}=\bar{w}-c_{f}, \quad \Lambda_{B}^{+}=c_{s}, \quad \Lambda_{B}^{-}=-c_{s}
$$

According to Proposition 2.4, the unknown matrices $R_{A}, R_{B}, T_{A}$, and $T_{B}$ must be chosen such that the conditions (18) and (19) are satisfied. There are different choices for these unknown matrices which lead to well-posedness.

One of the choices is the set of characteristic interface conditions

$$
R_{A}=[0,0], \quad T_{A}=\sqrt{c_{s}\left(c_{f}-\bar{w}\right)}, \quad R_{B}=0, \quad T_{B}=\left[0, \sqrt{c_{s}\left(\bar{w}+c_{f}\right)}\right],
$$

and $\alpha_{c}^{p}=1$. But the choices in (54) do not lead to the physical conditions (52) and (53). Hence, these conditions lack consistency. By inspection we find that the matrices

$$
\begin{array}{lll}
R_{A}=\left[0, \frac{\left(c_{f}-\bar{w}\right)\left(\bar{\rho} c_{f}-E / c_{s}\right)}{\bar{\rho} c_{f}+E / c_{s}}\right], & R_{B}=-\frac{c_{s}\left(\bar{\rho} c_{f}-E / c_{s}\right)}{\bar{\rho} c_{f}+E / c_{s}}, \\
T_{A}=\frac{2 E / c_{s}\left(c_{f}-\bar{w}\right)}{\bar{\rho} c_{f}+E / c_{s}}, & T_{B}=\left[0, \frac{2 c_{s} \bar{\rho} c_{f}}{\bar{\rho} c_{f}+E / c_{s}}\right]
\end{array}
$$

lead to

$$
w+p=v_{t}-E v_{x}, \quad \bar{\rho} c_{f} w+p=\bar{\rho} c_{f} v_{t}-E v_{x},
$$

which is equivalent to the physical conditions (52) and (53) and hence consistent. The choice (55) and $\alpha_{c}^{p}=\rho_{s}\left(c_{f}-\bar{w}\right) / \bar{\rho} c_{f}$ imply that the conditions (18) and (19) are satisfied, which means that the coupled problem (1)-(2) is well posed.

Finally, we also apply boundary conditions to the model problem. Since two eigenvalues of matrix $A$ are positive, two boundary conditions are needed at $x=-1$. The matrix $B$ has one negative eigenvalue, which means that one boundary condition is needed at $x=1$. We choose the general characteristic boundary conditions

$$
\left(X_{+}^{T}-R_{l} X_{-}^{T}\right) u(-1, t)=g_{l}(t), \quad\left(Y_{-}^{T}-R_{r} Y_{+}^{T}\right) v(1, t)=g_{r}(t),
$$

where $R_{l}$ and $R_{r}$ are matrices of appropriate size.

5.1. Numerical results. In the numerical calculations, we will use the manufactured solutions

$$
\begin{aligned}
& u_{1}(x, t)=\sin (6 \pi(x-t)), \quad u_{2}(x, t)=0, \quad u_{3}(x, t)=\cos (6 \pi(x-t)), \\
& v_{1}(x, t)=v_{2}(x, t)=e^{t / \lambda} \sin (8 \pi(x-t))
\end{aligned}
$$

with $\lambda=0.05$ and the parameters $\bar{w}=0.5, c_{f}=1, \bar{\rho}=1, \gamma=1.4, \rho_{s}=1$, and $c_{s}=1$. The manufactured solution will provide data for the forcing function, boundary conditions, and initial function. The rate of convergence is calculated as

$$
q_{u}=\ln \left(\frac{\left\|\mathbf{u}^{N_{1}}-\mathbf{u}\right\|_{P_{u} \otimes I}}{\left\|\mathbf{u}^{N_{2}}-\mathbf{u}\right\|_{P_{u} \otimes I}}\right) / \ln \left(\frac{N_{1}}{N_{2}}\right), \quad q_{v}=\ln \left(\frac{\left\|\mathbf{v}^{N_{1}}-\mathbf{v}\right\|_{P_{v} \otimes I}}{\left\|\mathbf{v}^{N_{2}}-\mathbf{v}\right\|_{P_{v} \otimes I}}\right) / \ln \left(\frac{N_{1}}{N_{2}}\right),
$$

where $\mathbf{u}$ and $\mathbf{v}$ are the analytical solutions and $\mathbf{u}^{N_{i}}$ and $\mathbf{v}^{N_{i}}$ are the corresponding numerical solutions with $N_{i}$ grid points. 
TABLE 1

Rate of convergence $q_{u}$ for $u=\left(u_{1}, u_{2}, u_{3}\right)$.

\begin{tabular}{lllll}
\hline \hline$N$ & $S B P 21$ & $S B P 42$ & $S B P 63$ & $S B P 84$ \\
\hline 20 & - & - & - & - \\
40 & 2.593 & 2.764 & 3.442 & 3.823 \\
80 & 2.102 & 3.146 & 4.264 & 4.956 \\
120 & 2.033 & 3.070 & 4.510 & 5.148 \\
180 & 2.014 & 3.035 & 4.550 & 5.160 \\
240 & 2.007 & 3.018 & 4.632 & 5.128 \\
\hline \hline
\end{tabular}

TABLE 2

Rate of convergence $q_{v}$ for $v=\left(v_{1}, v_{2}\right)$.

\begin{tabular}{lllll}
\hline \hline$N$ & $S B P 21$ & $S B P 42$ & $S B P 63$ & $S B P 84$ \\
\hline 20 & - & - & - & - \\
40 & 2.077 & 2.161 & 3.121 & 5.070 \\
80 & 2.024 & 2.849 & 4.197 & 4.939 \\
120 & 2.008 & 2.998 & 4.241 & 4.878 \\
180 & 2.003 & 3.032 & 4.301 & 4.849 \\
240 & 2.002 & 3.028 & 4.411 & 4.932 \\
\hline \hline
\end{tabular}

The time-integration in this section is done using the classical fourth order explicit Runge-Kutta scheme [5] with $C F L$ number $=0.01$. We choose the final time $T=1.0$ with time step $\Delta t=C F L \times \Delta x$, which makes the time-error negligible. The semidiscrete scheme was implemented using SBP operators $S B P 21, S B P 42, S B P 63$, and $S B P 84$, which gives a global accuracy of $2,3,4$, and 5 , respectively $[28,30]$. The results can be seen in Tables 1 and 2 .

5.2. Superconvergence of the functional. In this section, we investigate the impact of dual consistency. A dual consistent scheme is obtained by choosing the penalty matrices as in (31). Small changes in the coefficients of the penalty matrices in (31) lead to a stable but dual inconsistent scheme. In the dual inconsistent case, we choose

$$
\boldsymbol{\Sigma}_{L}=\frac{3}{2} X\left[\begin{array}{cc}
0 & 0 \\
0 & I_{A}^{-}
\end{array}\right], \quad \boldsymbol{\Sigma}_{R}=-\frac{3}{2} Y\left[\begin{array}{cc}
I_{B}^{+} & 0 \\
0 & 0
\end{array}\right],
$$

which leads to stability.

In Tables 3 and 4 , the rates of convergence of the functional

$$
J(w)=\int_{-1}^{0} u_{1}+u_{2}+u_{3} d x+\int_{0}^{1} v_{1}+v_{2} d x
$$

TABLE 3

Rate of functional convergence for the dual inconsistent discretization.

\begin{tabular}{lllll}
\hline \hline$N$ & $S B P 21$ & $S B P 42$ & $S B P 63$ & $S B P 84$ \\
\hline 20 & - & - & - & - \\
40 & 1.887 & 1.859 & 3.981 & 6.232 \\
80 & 1.963 & 2.829 & 4.544 & 4.567 \\
120 & 1.987 & 2.950 & 4.625 & 4.681 \\
180 & 1.994 & 2.978 & 4.631 & 4.777 \\
240 & 1.997 & 2.988 & 4.661 & 4.965 \\
\hline \hline
\end{tabular}

Copyright $\odot$ by SIAM. Unauthorized reproduction of this article is prohibited. 
TABLE 4

Rate of functional convergence for the dual consistent discretization.

\begin{tabular}{lllll}
\hline \hline$N$ & $S B P 21$ & $S B P 42$ & $S B P 63$ & $S B P 84$ \\
\hline 20 & - & - & - & - \\
40 & 2.303 & 2.464 & 6.541 & 5.331 \\
80 & 2.077 & 4.101 & 6.123 & 7.439 \\
120 & 2.024 & 4.269 & 6.101 & 8.400 \\
180 & 2.010 & 4.256 & 6.022 & 8.533 \\
240 & 2.005 & 4.218 & 6.010 & 8.644 \\
\hline \hline
\end{tabular}

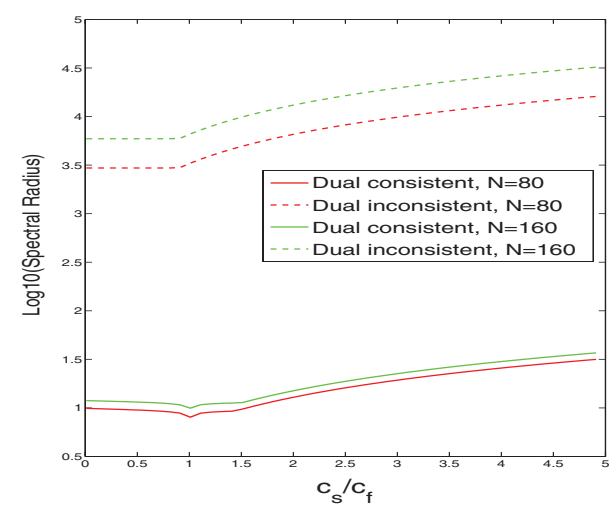

(a) $S B P 21$

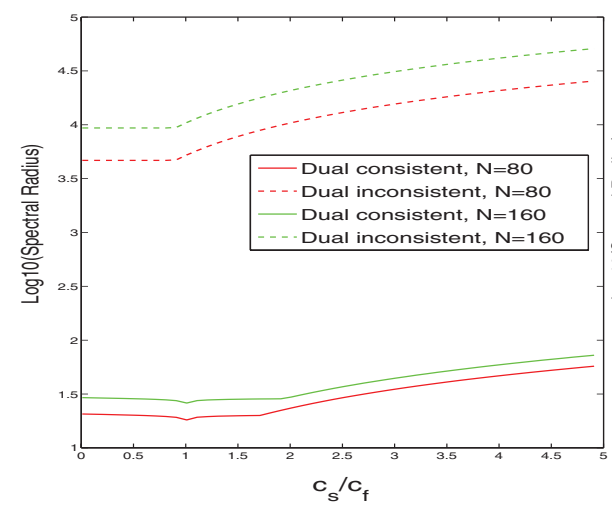

(c) $S B P 63$

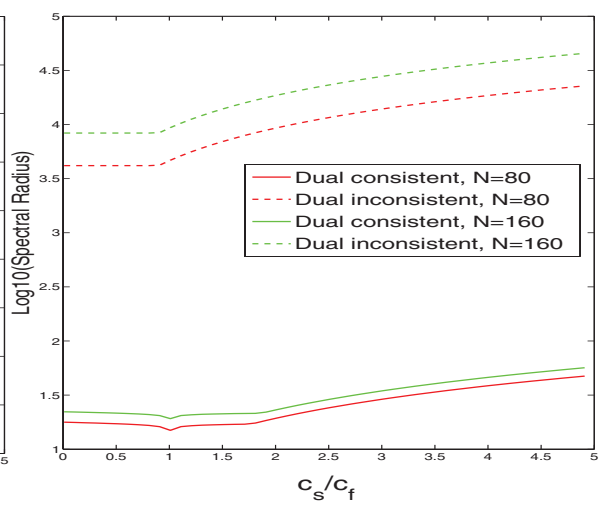

(b) $S B P 42$

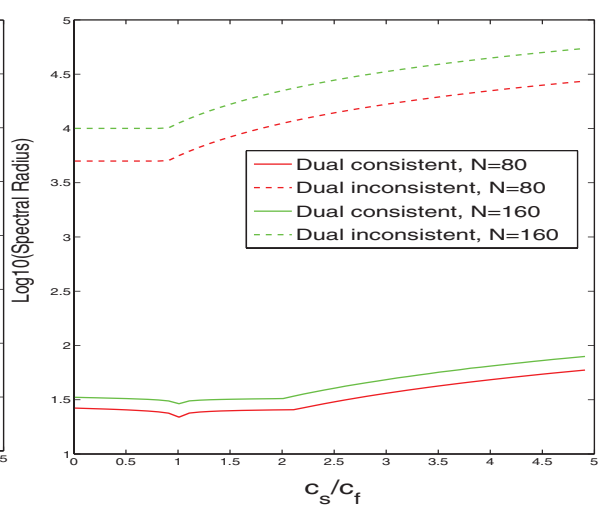

(d) SBP84

FIG. 1. Spectral radius of the semidiscrete approximation problem (56) for different operators and numbers of grid points.

are calculated and clear superconvergence can be seen. The superconvergent functional is obtained without requiring any knowledge about the solution of the dual equations. In order to have a dual consistent scheme, we only need the dual equation and its interface conditions, which are obtained by knowing the interface conditions of the primal problem. This means that superconverging functionals are obtained at no extra computational cost [2].

5.3. Stiffness. In practice, the wave speeds between fluid $\left(c_{f}\right)$ and solid $\left(c_{s}\right)$ are different, which may cause stiffness. We will check the stiffness of coupled problem (1)-(2) by computing the spectral radius of the resulting semidiscrete formulation

$$
\mathbf{w}_{t}=K \mathbf{w}+F .
$$

Copyright $@$ by SIAM. Unauthorized reproduction of this article is prohibited. 
In (56), $\mathbf{w}=[\mathbf{u}, \mathbf{v}]^{T}$ and the matrices $K$ and $F$ contain the complete spatial discretization including the coupling terms and the boundary data, respectively. Figure 1 shows the spectral radius of matrix $K$ for dual consistent and dual inconsistent cases. Clearly, the dual consistent scheme leads to smaller spectral radius and less stiffness. One can also see that the stiffness increases as $c_{s} / c_{f}$ increases above one.

6. Summary and conclusions. We have considered the coupling of two general hyperbolic systems. The energy method was used to derive general well posed interface conditions. It was shown that the derived interface conditions lead to a well posed problem for both weak and strong imposition.

The equations were discretized using finite differences of SBP-SAT form. The penalty matrices were derived in the analysis of the continuous problem. Almost no additional derivations were necessary.

Next, the dual problem and its well posed interface conditions were derived using the energy method. The interface conditions were imposed weakly and strongly also for the dual problem. The weak interface procedures lead directly to stability of the numerical approximation as in the primal problem. The numerical scheme was shown to be dual consistent for specific choices of the penalty matrices.

By considering a physical example, it was shown that the mathematical interface conditions contain the physically correct interface conditions. The mathematical theory can also narrow the search for well posed and accurate interface conditions.

The rate of convergence was verified by the method of manufactured solution and the result was consistent with the SBP-SAT theory. Superconverging functionals were obtained for dual consistent discretizations. It was also found that dual consistency reduced the stiffness of the discretizations.

\section{REFERENCES}

[1] S. Abarbanel and D. Gottleib, Optimal time splitting for two- and three-dimensional Navier-Stokes equations with mixed derivatives, J. Comput. Phys., 41 (1981), pp. 1-43.

[2] J. Berg And J. Nordström, Superconvergent functional output for time-dependent problems using finite differences on summation-by-parts form, J. Comput. Phys., 231 (2012), pp. 6846-6860.

[3] J. Berg AND J. Nordström, On the impact of boundary conditions on dual consistent finite difference discretizations, J. Comput. Phys., 236 (2013), pp. 41-55.

[4] J. Berg And J. Nordström, Duality based boundary conditions and dual consistent finite difference discretizations of the Navier-Stokes and euler equations, J. Comput. Phys., 259 (2014), pp. 135-153.

[5] J. C. Butcher, Coefficients for the study of runge-kutta integration processes, J. Aust. Math. Soc., 3 (1963), pp. 185-201.

[6] M. Carpenter, J. Nordström, and D. Gottlieb, A stable and conservative interface treatment of arbitrary spatial accuracy, J. Comput. Phys., 148 (1999), pp. 341-365.

[7] M. H. Carpenter, D. Gottlieb, and S. Abarbanel, Time-stable boundary conditions for finite-difference schemes solving hyperbolic systems: Methodology and application to highorder compact schemes, J. Comput. Phys., 111 (1994), pp. 220-236.

[8] B. Flemisch, M. Kaltenbacher, And B. I. Wohlmuth, Elasto-acoustic and acousticacoustic coupling on nonmatching grids, Internat. J. Numer. Methods Engrg., 67 (2006), pp. 1791-1810.

[9] B. Gustafsson, H.-O. Kreiss, and J. Oliger, Time Dependent Problems and Difference Methods, Wiley Interscience, New York, 1995.

[10] J. E. HiCKEN AND D. W. ZINGG, Superconvergent functional estimates from summation-byparts finite-difference discretizations, SIAM J. Sci. Comput., 33 (2011), pp. 893-922.

[11] R. A. Horn And C. R. Johnson, Topics in Matrix Analysis, Cambridge University Press, Cambridge, UK, 1991.

[12] X. Huan, J. E. Hicken, AND D. W. ZINGG, Interface and boundary schemes for high-order methods, in Proceedings of the 19th AIAA Fluid Dynamics Conference, San Antonio, TX, 2009, pp. 22-25. 
[13] A. Jameson, Aerodynamic design via control theory, J. Sci. Comput., 3 (1988), pp. 233-260.

[14] Y. JARny, M. N. Ozisik, AND J. P. BARdon, A general optimization method using adjoint equation for solving multidimensional inverse heat conduction, Int. J. Heat Mass Transfer, 34 (1991), pp. 2911-2919.

[15] H.-O. Kreiss, Problems with different time scales for partial differential equations, Comm. Pure Appl. Math., 33 (1980), pp. 399-439.

[16] S. Li And L. Petzold, Adjoint sensitivity analysis for time-dependent partial differential equations with adaptive mesh refinement, J. Comput. Phys., 198 (2004), pp. 310-325.

[17] J. Lindström And J. NoRdSTRÖm, A stable and high-order accurate conjugate heat transfer problem, J. Comput. Phys., 229 (2010), pp. 5440-5456.

[18] K. Mattsson, Boundary procedures for summation-by-parts operators, J. Sci. Comput., 18 (2003), pp. 133-153.

[19] B. Muha AND S. ČAnIĆ, Existence of a solution to a fluid-multi-layered-structure interaction problem, J. Differential Equations, 256 (2014), pp. 658-706.

[20] A. J. Mulholland, R. Picard, S. Trostorff, and M. Waurick, On well-posedness for some thermo-piezoelectric coupling models, Math. Methods Appl. Sci., 39 (2016), pp. 4375-4384.

[21] J. Nordström, A roadmap to well posed and stable problems in computational physics, J. Sci. Comput., (2016), pp. 1-21.

[22] J. Nordström And J. Berg, Conjugate heat transfer for the unsteady compressible NavierStokes equations using a multi-block coupling, Comput. \& Fluids, 72 (2013), pp. 20-29.

[23] J. Nordström AND S. ERIKSSOn, Fluid structure interaction problems: The necessity of a well posed, stable and accurate formulation, Commun. Comput. Phys., 8 (2010), pp. 1111-1138.

[24] J. Nordström, J. Gong, E. van der Weide, And M. Svärd, A stable and conservative high order multi-block method for the compressible Navier-Stokes equations, J. Comput. Phys., 228 (2009), pp. 9020-9035.

[25] J. Nordström and M. SvÄrd, Well-posed boundary conditions for the Navier-Stokes equations, SIAM J. Numer. Anal., 43 (2005), pp. 1231-1255.

[26] B. Roe, R. Jaiman, A. Haselbacher, and P. H. Geubelle, Combined interface boundary condition method for coupled thermal simulations, Internat. J. Numer. Methods Fluids, 57 (2008), pp. 329-354.

[27] B. SJÖGREEN AND J. W. BANKs, Stability of finite difference discretizations of multi-physics interface conditions, Commun. Comput. Phys., 13 (2012), pp. 386-410.

[28] B. Strand, Summation by parts for finite difference approximations for $d / d x$, J. Comput. Phys., 110 (1994), pp. 47-67.

[29] M. SvÄRD, M. CARPENTER, AND J. NoRdStröm, A stable high-order finite difference scheme for the compressible Navier-Stokes equations: Far-field boundary conditions, J. Comput. Phys., 225 (2007), pp. 1020-1038.

[30] M. SVÄRD AND J. NORDSTRÖM, On the order of accuracy for difference approximations of initial-boundary value problems, J. Comput. Phys., 218 (2006), pp. 333-352.

[31] M. SvÄRD And J. Nordström, A stable high-order finite difference scheme for the compressible Navier-Stokes equations: No-slip wall boundary conditions, J. Comput. Phys., 227 (2008), pp. $4805-4824$.

[32] M. SvÄRD AND J. NORDSTRÖM, Review of summation-by-parts schemes for initial-boundaryvalue problems, J. Comput. Phys., 268 (2014), pp. 17-38.

[33] K. Virta And K. Mattsson, Acoustic wave propagation in complicated geometries and heterogeneous media, J. Sci. Comput., 61 (2014), pp. 90-118.

Copyright (c) by SIAM. Unauthorized reproduction of this article is prohibited. 